\title{
Identification of $M Y B$ transcription factor genes and their expression during abiotic stresses in maize
}

\author{
Y.H. CHEN ${ }^{1}$, Y.Y. CAO ${ }^{1}$, L.J. WANG ${ }^{1}$, L.M. $\mathrm{LI}^{1}$, J. YANG ${ }^{1}$, and M.X. ZOU ${ }^{2}$ \\ School of Life Science ${ }^{1}$ and School of Public Health ${ }^{2}$, Nantong University, Jiangsu, 226019, P.R.China
}

\begin{abstract}
The $M Y B$ transcription factor superfamily is a large gene family that plays central roles in developmental processes and defence responses in plants. Unlike in Arabidopsis, only few members of the R2R3-MYB gene family have been functionally well characterized in maize, especially in abiotic stress-response pathways. Subgroup-specific conserved motifs outside the MYB domain may reflect functional conservation. A comparative genomics study using Arabidopsis abiotic stress-responsive MYB protein sequences identified 46 ZmMYB genes that may be involved in abiotic stress responses of Zea mays. An expression pattern analysis of the $46 \mathrm{ZmMYB}$ genes under abiotic stress treatments was used to identify $22 M Y B$ genes that were induced by one or more of the stress treatments. ZmMYB30 was highly up-regulated under the four stress treatments. The ectopic expression of ZmMYB30 in transgenic Arabidopsis plants promoted saltstress tolerance and also increased the expression of a number of abiotic stress-related genes, allowing the plants to overcome adverse conditions.
\end{abstract}

Additional key words: abscisic acid, low temperature, osmotic stress, salinity, Zea mays.

\section{Introduction}

Abiotic stresses, such as drought, salinity, and cold, negatively affect vegetative and reproductive growth and the productivity of plants, including maize (Habben et al. 2014, Harrison et al. 2014, Lobell et al. 2014). Therefore, the identification and characterization of key genes that mediate maize responses to abiotic stresses, as well as the study of the molecular mechanisms involved, will provide important information necessary to engineer or select maize having an enhanced tolerance to abiotic stresses (Hoang et al. 2014).

In recent years, several proteins that play roles in maize abiotic stress resistance, especially in drought resistance, have been identified. These proteins have been classified into two major categories, transcription factors, such as ZmbZIP72, ZmWRKY33, and ZmSNAC1, and protein kinases, such as ZmMPK17, ZmCPK4, and ZmMKK1(Lu et al. 2012, Pan et al. 2012, Ying et al. 2012, Jiang et al. 2013, Li et al. 2013, Cai et al. 2014,
Zhang et al. 2014, Zhou et al. 2016).

Transcription factors (TFs) are major players in regulating plant responses against adverse abiotic factors (Hoang et al. 2014). Among them, the large MYB family participates in plant responses to various stressors as was verified in model plant species, such as Arabidopsis thaliana and rice (Li et al. 2015). In addition to the MYB proteins in model species, several other MYB proteins are involved in drought responses in crops. In wheat, TaPIMP1 is up-regulated during drought stress, and transgenic tobacco expressing this gene displays enhanced tolerance (Zhang et al. 2012b). The expression of TaMYB30 and its homologs is induced in response to polyethylene glycol (PEG) stress, and the over-expression of TaMYB30-B, one of three homologs, improves the drought tolerance of transgenic Arabidopsis (Zhang et al. 2012a). In cotton, GbMYB5 is positively involved in plant adaptation to drought stress (Chen et al. 2015).

Submitted 28 October 2016, last revision 26 May 2017, accepted 14 June 2017.

Abbreviations: ABA - abscisic acid; CaMV - cauliflower mosaic virus; DBD - DNA binding domain; ML - maximum likelihood; MS - Murashige and Skoog; NJ - neighbor-joining; ORFs - open reading frames; PEG - polyethylene glycol; qPCR - quantitative PCR; TFs - transcription factors; WT - wild type.

Acknowledgments: This study was supported by grants from the Jiangsu Overseas Research \& Training Program for University Prominent Young \& Middle-aged Teachers and Presidents, the Science and Technology Fund of Nantong City (MS12015091), and the Undergraduate Innovative Training Program of Nantong University.

* Corresponding author; e-mail: chenyh@ntu.edu.cn 
However, less is known about the function of the MYB family in maize. Using a genomic comparative analysis, 157 R2R3-MYB genes were identified in maize (Du et al. 2012), but the stress-response-related $M Y B$ genes have not been determined until now.

The aim of this study was to identify the stressresponse-related $M Y B$ genes and we used the following three approaches: 1) comparative analysis to reveal potential stress response related $M Y B$ genes; 2) expression pattern analysis on these potential genes under stress treatment to find candidate genes; and 3) functional analysis on the candidate gene(s). This first comprehensive study of the involvement of the $M Y B$ gene family in maize stress responses could provide valuable data for the further exploration of the functions of this significant gene family in this plant.

\section{Materials and methods}

Plants, growth conditions, and stress treatments: The seeds of maize (Zea mays L. cv. B73) were germinated on moistened gauze under dark at $28{ }^{\circ} \mathrm{C}$. The seedlings were then transferred to Hoagland's liquid medium to grow at a temperature of $28^{\circ} \mathrm{C}$, an air humidity of $70 \%$, a 16-h photoperiod, and an irradiance of $120 \mu \mathrm{mol} \mathrm{m}^{-2} \mathrm{~s}^{-1}$. Seedlings at the three-leaf stage were used in subsequent experiments. To follow effects of high salinity, drought, and ABA, the seedlings were treated with $200 \mathrm{mM} \mathrm{NaCl}$, $20 \%(\mathrm{~m} / \mathrm{v})$ polyethylene glycol (PEG6000), and $100 \mu \mathrm{M}$ ABA, respectively. The third-leaf tissues were collected at $0,3,6,12,24$, and $48 \mathrm{~h}$. For the cold treatment, threeleaf stage seedlings were transferred from 28 to $6{ }^{\circ} \mathrm{C}$, and the leaf samples were harvested at $0,3,6,12,24$, and $48 \mathrm{~h}$. The stress-treatment experiments were repeated three times.

Identification of putative abiotic stress related $M Y B$ genes in maize: There are many abiotic stress related MYB genes in other species, such as Arabidopsis, rice, and wheat. Using the amino acid sequences of these MYBs as query sequences (a total of 35 proteins; see Table 1 Suppl.); we performed a search with the BLASTP algorithm on the NCBI website (http://blast.ncbi.nlm. nih.gov/Blast.cgi?PAGE=Proteins). Maize (taxid: 4577) was selected in the organism column. The query results that were greater than $57 \%$ in query coverage column were selected for further analyses. All of the BLASTP results were screened using the following procedure: firstly, the redundant MYB proteins and proteins with incomplete open reading frame were discarded; and secondly, all of the proteins were subjected to multiple alignment analyses by ClustalW (www.ebi.ac.uk/ clustalw/) and a phylogenetic tree analysis to exclude the MYB proteins that did not fit the tree. To verify the reliability of our results, the functional and structural domains of these candidate maize MYB proteins were predicted using PROSITE profiling (http://www. expasy.org/tools/scanprosite/) and SMART analysis (http://smart.embl-heidelberg.de/).

Phylogenetic analysis: The amino acid sequences of Arabidopsis (21 R2R3-MYB members), maize (46 R2R3-MYB members), and 14 other MYB proteins from other species, including rice and wheat, were downloaded from TAIR and NCBI databases. The names and amino acid sequences of the proteins are listed in Table 1 Suppl. The complete amino acid sequences of the MYB proteins were used to construct phylogenetic trees. Sequence alignments were performed with ClustalW using the web platform (https://npsa-prabi.ibcp.fr/cgibin/npsa_automat.pl?page=/NPSA/npsa_clustalw.html). A neighbor-joining (NJ) tree and maximum likelihood (ML) tree were constructed individually using the MEGA6 program (Tamura et al. 2013), and internal branch support was estimated with 1000 bootstrap replicates.

Conservative analysis of potential protein motifs within and outside the maize R2R3-MYB DNA-binding domains (DBDs): To analyze the features of the MYB domain of the stress-responsive R2R3-MYB proteins, the sequences of the R2 and R3 MYB repeats in the 57 R2R3-MYB proteins (22 from maize, 21 from Arabidopsis, and 14 proteins from other species) were aligned with the ClustalW method using BiOEdit software and adjusted manually. The sequence logos for the R2 and R3 MYB repeats were obtained by submitting the multiple alignment sequences to the website http://weblogo.berkeley.edu/logo.cgi. To identify potential protein motifs outside the maize MYB domain, the full amino acid sequences of 57 proteins were analyzed using the motif discovery software $M E M E$ Suite 4.11.1 (http://meme-suite.org/tools/meme), with the following parameter settings: the distribution of motifs, 0 or 1 per sequence; maximum number of motifs to find, 20; minimum width of motif, 6; maximum width of motif, 50; and the motif must be present in all members within the same subgroup. In addition, only motifs with e-value \#1e-10 were kept for further analyses. Subsequently, the MAST program was used to search for detected motifs in protein databases.

Real-time quantitative PCR (qPCR) analysis: Total RNA was extracted using TRIzol reagent from leaves of maize and Arabidopsis thaliana L. (cv. Columbia, 30-dold) and treated with DNase I. For each sample, $3 \mu \mathrm{g}$ of total RNA was used to synthesize first-strand cDNA with SuperScript ${ }^{\mathrm{II}}$ reverse transcriptase (Invitrogen, Waltham, USA). For real-time qPCR, each reaction contained 
$10 \mathrm{~mm}^{3}$ of $2 \times$ SYBR Green PCR Master Mix (Roche, Basel, Switzerland), $2.0 \mathrm{~mm}^{3}$ of cDNA (10-fold dilution), and $1 \mathrm{~mm}^{3}$ of gene-specific primers $(2.0 \mu \mathrm{M})$ in a final volume of $20 \mathrm{~mm}^{3}$. The thermal cycling condition were $95{ }^{\circ} \mathrm{C}$ for $2 \mathrm{~min}$; followed by 40 cycles at $95{ }^{\circ} \mathrm{C}$ for $20 \mathrm{~s}$, at $57{ }^{\circ} \mathrm{C}$ for $30 \mathrm{~s}$, and at $72{ }^{\circ} \mathrm{C}$ for $31 \mathrm{~s}$. The maize and Arabidopsis Actin genes were used as references, and the reactions were performed using the ABI Prism 7000 realtime PCR system (Applied Biosystems, Waltham, USA). The reactions were repeated three times, with three biological replicates each. The quantitative analysis used the $2^{-} \Delta \Delta^{\mathrm{Ct}}$ method. The gene-specific primers for the 46 maize $M Y B$ genes and Arabidopsis stress-responsive genes can be found in Table 4 Suppl.

Transformation of $Z \mathbf{m M Y B 3 0}$ in Arabidopsis: The fulllength coding sequence (ATG to TAA) of ZmMYB30 was amplified from maize leaf cDNA by PCR using primers 5'-GAAGGTACCATGGCGTCGTCTTCTCCGTCCTG-3' and 5'-AGGTCTAGACTACTCGATCTTGGCCAT ACCCATC-3', which contain KpnI and XbaI enzyme digestion sites. The PCR product was inserted in the pCAMBIA 1300 vector with a $35 \mathrm{~S}$ cassette $(35 \mathrm{~S}$ enhancer $+35 \mathrm{~S}$ promoter + multiple cloning site + terminator) by $K p n \mathrm{I}$ and $X b a \mathrm{I}$ enzyme digestion. The construct was confirmed by sequencing and then transformed into

\section{Results}

In general, orthologous proteins from different species share similar structures and functions. To uncover the putative abiotic stress-responsive MYB TF(s) in maize, we used full length sequences of well-known abiotic stress-response-related MYB proteins from Arabidopsis, wheat, and rice as queries (Table 1 Suppl.), and a BLASTP algorithm-based search was performed to reveal the maize MYB proteins having high similarities. After discarding the redundant protein and the incomplete open reading frame-containing proteins, 46 maize R2R3MYBs were identified. Using the neighbor-joining method in MEGA6 and the ML method in PhyML, we performed a phylogenetic reconstruction containing the Arabidopsis defence-related R2R3-MYBs (21 members), 14 well-characterized R2R3-MYBs from other plant species and their 46 orthologs in maize. The topology of phylogenetic trees was illustrated in Fig. 1 Suppl.

The phylogenetic comparative analysis of these abiotic stress-related R2R3-MYBs (putative in maize) in different plant species revealed considerable diversification and conservation. In the phylogenetic tree, there are nine clades and most of the clades contained members of orthologous genes belonging to maize, Arabidopsis, and/or other plant species, such as Clades 6, 7, and 9. However, Clades 3 and 8 are maize specific, having only maize genes.
Agrobacterium strain GV3101. The Arabidopsis ZmMYB30 ectopic expression transgenic lines (OE) were generated using an Agrobacterium-mediated transformation protocol and screened using $20 \mathrm{mg} \mathrm{dm}^{-3}$ hygromycin (Roche). The empty vector was also transformed into Arabidopsis (Columbia ecotype) to produce control transgenic $(\mathrm{CK})$ plants.

Salt treatment of transgenic Arabidopsis plants: The surface-sterilized seeds from three OE lines (OE-1, OE-2, OE-3), CK, and wild-type plants were sown on Murashige and Skoog (MS) medium-containing plates with $1 \%(\mathrm{~m} / \mathrm{v})$ sucrose and $0.8 \%(\mathrm{~m} / \mathrm{v})$ agar, and MS plates independently supplemented with 50, 100, and $150 \mathrm{mM} \mathrm{NaCl}$. The plates were placed at $4{ }^{\circ} \mathrm{C}$ for $2 \mathrm{~d}$ and then moved to a vertical position under a 16-h photoperiod, irradiance of $120 \mu \mathrm{mol} \mathrm{m} \mathrm{m}^{-2}$, and a temperature of $22{ }^{\circ} \mathrm{C}$. Three days after growth in the control and salt-treated media, the percentages of radicle emergence were carefully counted to describe the seed germination rates. The root lengths of the seedlings were measured in the germinated lines after being grown vertically on normal media for $7 \mathrm{~d}$ and then transferred to the salt media. The root lengths were recorded $3 \mathrm{~d}$ after being transferred. The salt-treatment experiment was repeated three times.
The expression profiles of the 46 maize $M Y B$ genes under different abiotic stresses $(200 \mathrm{mM} \mathrm{NaCl}, 20 \%$ PEG6000, $6^{\circ} \mathrm{C}$, and $100 \mu \mathrm{M}$ ABA) were screened to find stress-induced genes using the real-time PCR. As a whole, 22 genes were detected that responded to at least one treatment, including 14 genes that responded to exogenous ABA (Fig. 1), 6 genes that responded to low temperature (Fig. 2), 14 genes that responded to $\mathrm{NaCl}$ (Fig. 3), and 13 genes that responded to PEG (Fig. 4). Among these genes, 16 responded to more than one treatment (Table 3 Suppl.). For example, ZmMYB30 was induced by four kinds of treatments, while ZmMYB53 was only induced by cold stress. In the ABA-, PEG- and salt-stress treatments, the expressions of most genes peaked 12 or $24 \mathrm{~h}$ after treatment and then decreased; while in the cold treatment, the expression patterns of ZmMYB30, ZmMYB53, ZmMYB95 and ZmMYB139 were different, with the peak values being delayed until $48 \mathrm{~h}$.

After the expression pattern analysis, the amino acid sequences of 22 stress-induced ZmMYB proteins and 35 MYB proteins involved in stress response from other species were entered in the query box to determine any conserved motifs using $M E M E$. In the DBD, similar to counter-parts in other plant species, the basic regions of these 22 maize R2R3-MYB domains contained on average 108 basic residues with a rare frequency of 

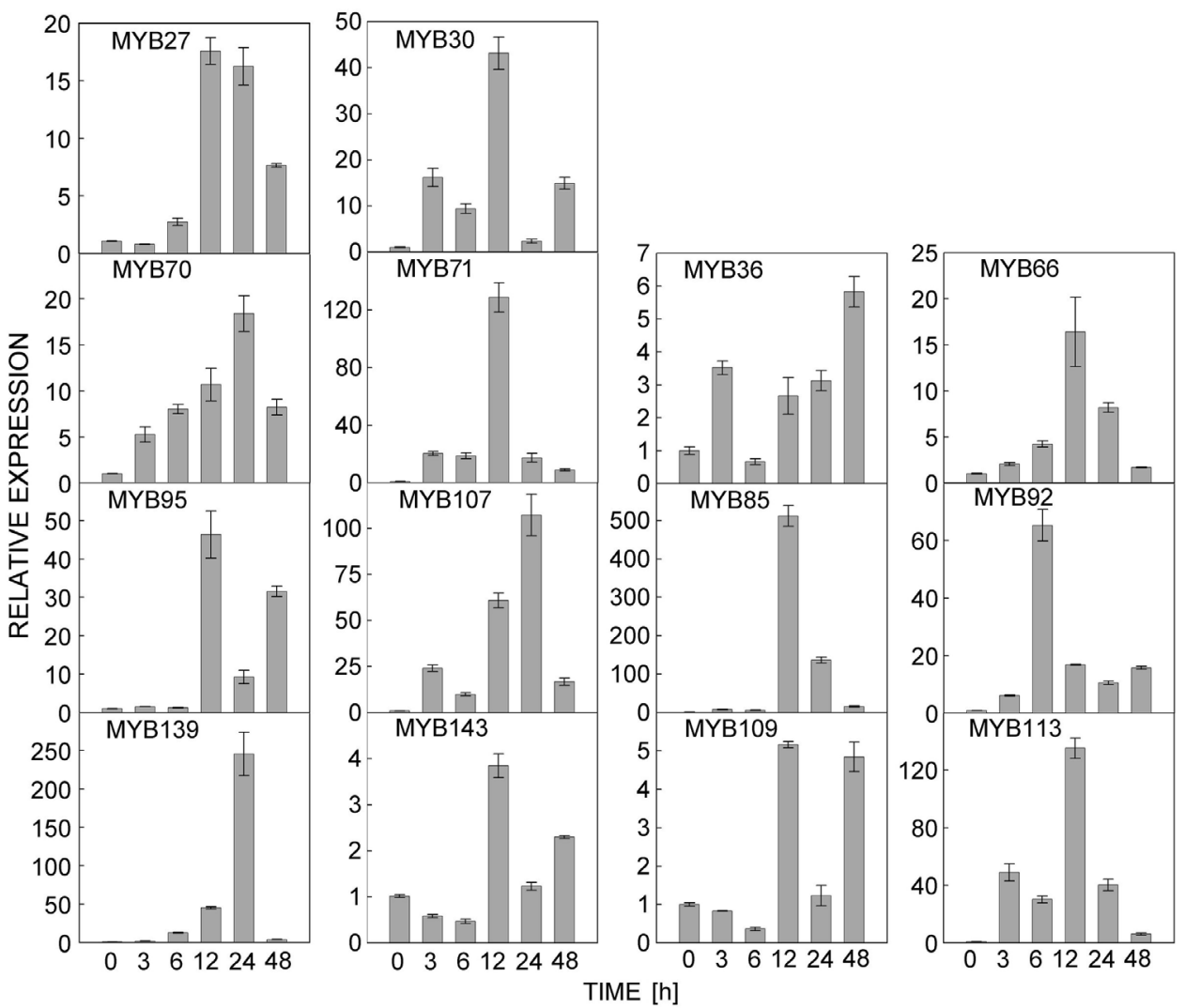

Fig. 1. Expression patterns of 14 ABA-responsive $Z m M Y B$ genes under $100 \mu \mathrm{M}$ ABA treatment. The ABA-responsive $Z m M Y B$ genes were identified by real-time qPCR and Actin gene was used as reference. Maize seedlings without treatment was referred as control $(0 \mathrm{~h})$ and set to 1 . Means $\pm \mathrm{SD}, n=3$.

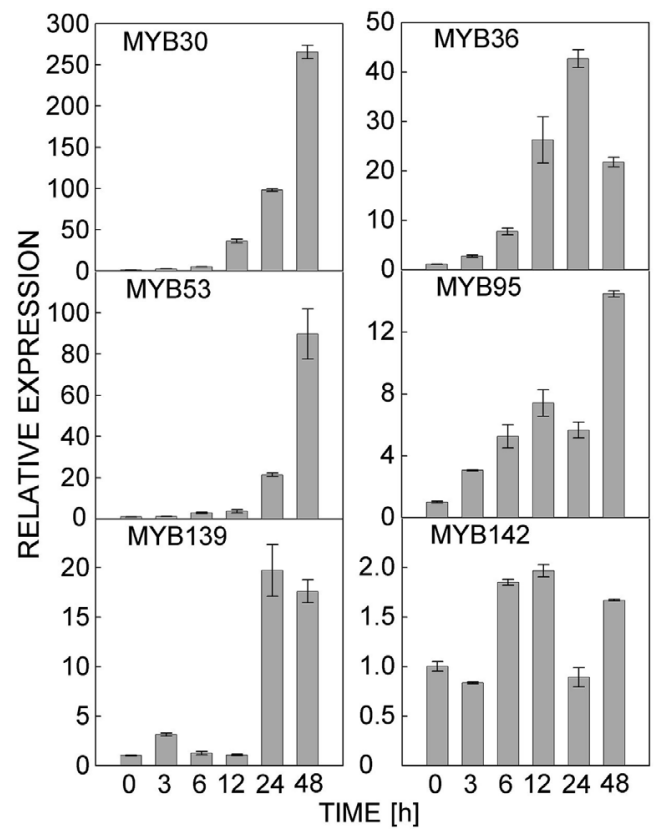

Fig. 2. Expression patterns of 6 cold-responsive $Z m M Y B$ genes under low temperature $\left(6^{\circ} \mathrm{C}\right)$ treatment. For other detail see Fig. 1. deletion or insertion $(>2 \%)$. On the contrary, the region outside the the DBD was the most divergent, both in protein length and amino acid composition. The R2 and R3 MYB repeats had similar structures (Fig. 6), including a series of evenly distributed and highly conserved tryptophan (Trp/W) residues (Fig. 2 Suppl.). The five Trp residues, including three in the R2 repeat and two in the $\mathrm{R} 3$ repeat at particular positions, are highly conserved in plant MYB' DBDs. In addition to the conserved Trp sites, many other residues were also highly conserved.

In the C-termini of these MYB proteins, other conserved motifs with variable lengths (6 - 50 amino acids) were detected. Seven motifs were identified, and three motifs had more than six MYB protein members (Fig. 2 Suppl.). Motifs 2 and 4 had 10 members, and motif 6 had 6 members. The proteins sharing a motif are always classified into the same subgroup. For instance, 10 MYB proteins (AtMYB70, AtMYB44, ZmMYB085, TaMYB1, ZmMYB142, AtMYB77, AtMYB73, ZmMYB095, ZmMYB030, and ZmMYB109) shared the C-terminal Motif 2 (Fig. 2 Suppl.) from the same clade 2 of the phylogenetic tree (Fig. 1 Suppl.). These subgroupspecific motifs may provide additional functional 
Y.H. CHEN et al.
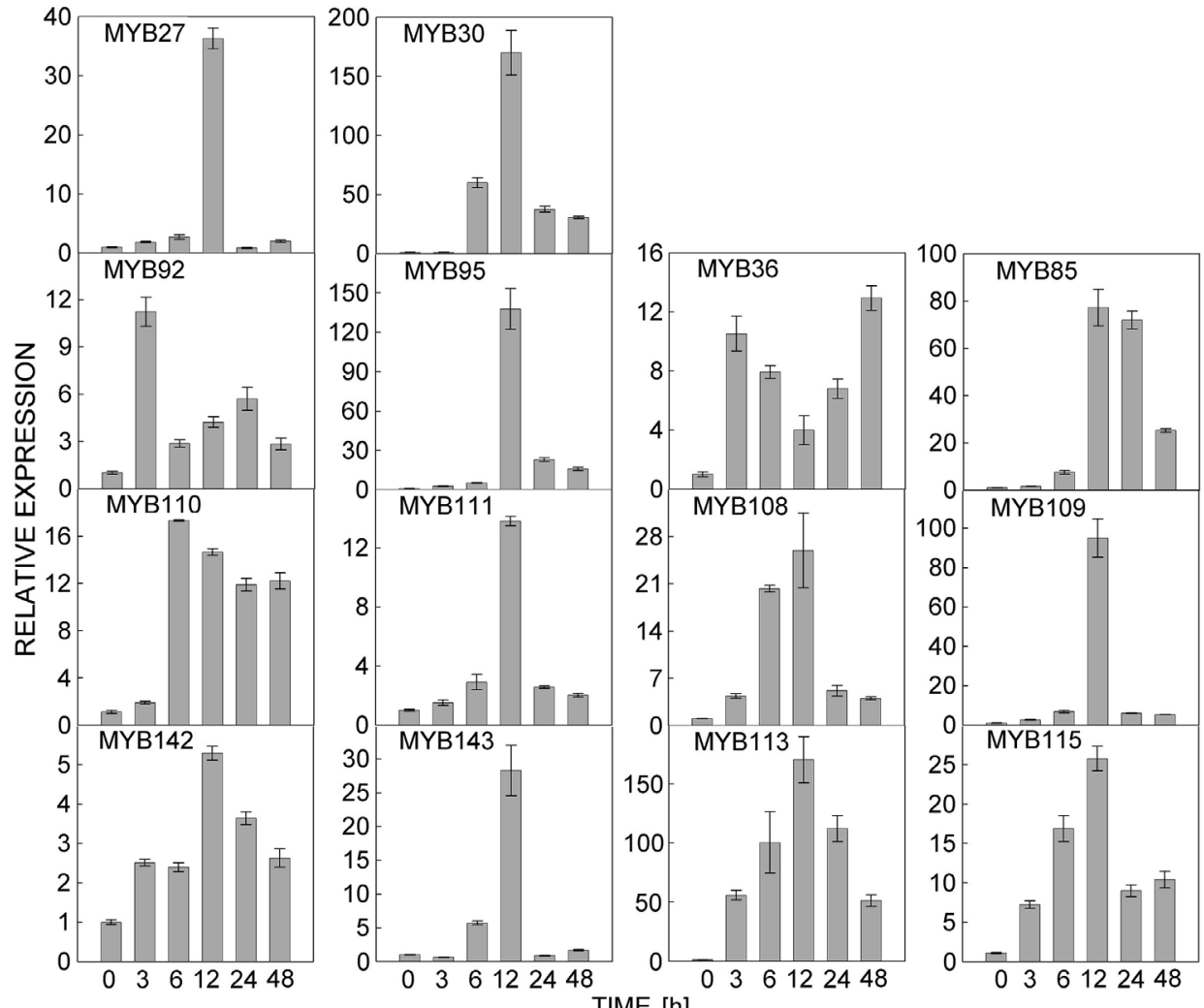

Fig. 3. Expression patterns of 14 salt-responsive $Z m M Y B$ genes under salt treatment ( $200 \mathrm{mM} \mathrm{NaCl})$. For other detail see Fig. 1.
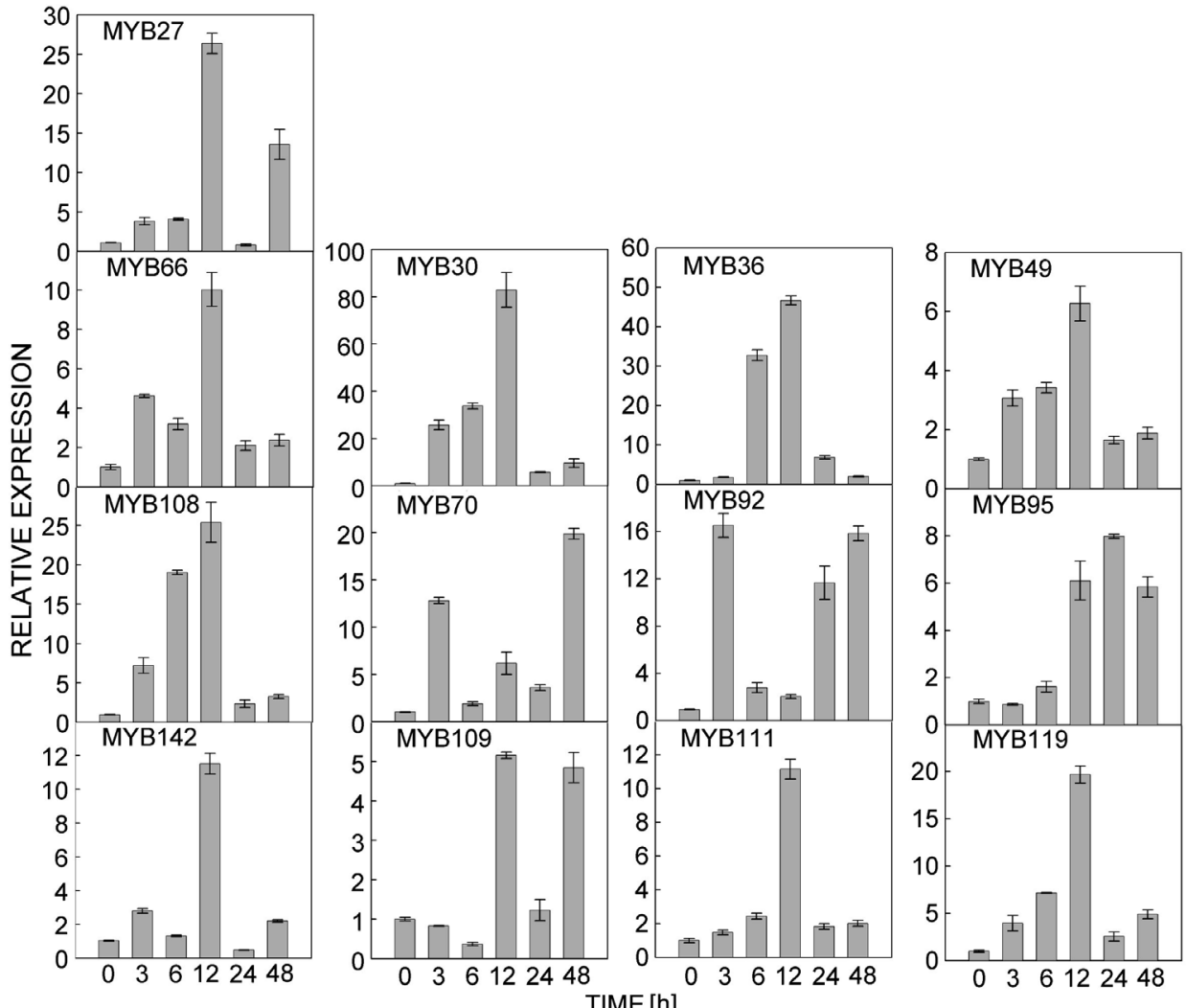

Fig. 4. Expression patterns of 13 drought-responsive ZmMYB genes under osmotic stress (20\% PEG). For other detail see Fig. 1. 


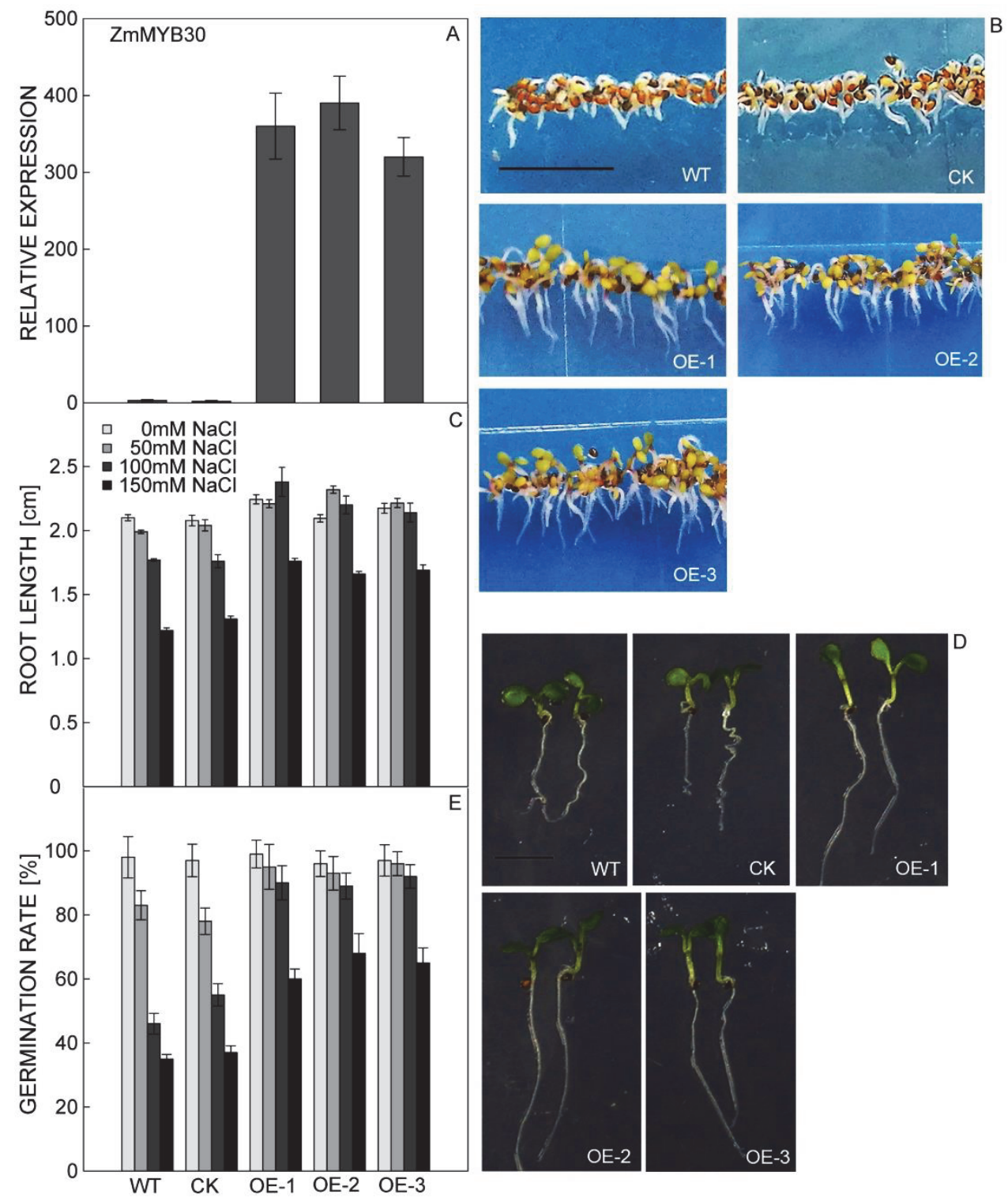

Fig. 5. $A$ - The expression of ZmMYB30 in three ectopic transgenic Arabidopsis lines (OE1, OE2, and OE3), control (CK), and wild type (WT) plants determined by real-time qPCR. $B$ to $E$ - Germination and growth of WT, CK, OE1, OE2, and OE3 on MS media with $\mathrm{NaCl}(B$ - after growth on $100 \mathrm{mM} \mathrm{NaCl}$ for $4 \mathrm{~d}$, bar $=1 \mathrm{~cm} ; C, E$ - root length and germination rate on media with different $\mathrm{NaCl}$ concentrations; $D$ - seedlings after growth on MS medium for $7 \mathrm{~d}$ and then transferred to MS medium containing $100 \mathrm{mM} \mathrm{NaCl}$ for another $3 \mathrm{~d}, b a r=0.5 \mathrm{~cm})$. Means $\pm \mathrm{SDs}, n=3$.

properties and enabled us to determine the regulatory specificity of the proteins.

To determine the functions of maize $M Y B$ genes in plant abiotic stress responses, we selected the ZmMYB30 gene for further experimental analysis because it was induced under all four treatments and its expression was higher than that of other genes. ZmMYB30 was ectopicexpressed in Arabidopsis under the control of the cauliflower mosaic virus $35 S$ promoter. Using a real-time PCR analysis, we found that the expression of ZmMYB30 in the three OE lines was much higher than in the control plant (Fig. 5A). For the salt-tolerance analysis of $\mathrm{OE}$, three transgenic lines of $\mathrm{T} 2$ progeny (OE-1, OE-2, and
OE-3), CK, and wild type (WT) plants were grown under normal conditions and high salinity $(50,100$, and $150 \mathrm{mM} \mathrm{NaCl}$ ). Under normal conditions, no obvious differences in the phenotypes among WT, CK, and OE seedlings were observed. All of the plants germinated simultaneously and there were no differences among the root lengths. When plants were grown on MS plates containing 50, 100 and $150 \mathrm{mM} \mathrm{NaCl}$, the three transgenic $\mathrm{OE}$ seeds germinated earlier than both WT and CK seeds (Fig. 5B,C). The roots lengths of all three $\mathrm{OE}$ transgenic seedlings were longer than those of the CK and WT plants (Fig. 5D,E). These results indicate that the ectopic expression of ZmMYB30 enhanced the salt 
tolerance in Arabidopsis. To investigate the molecular mechanism underlying the responses of OE plants to salt stress, the expression of 14 known stress-responsive genes in $\mathrm{OE}$ plants grown under normal conditions were examined by real-time qPCR. Compared with the $\mathrm{CK}$ plants, eight genes, DREB2A, RD20, ABI5, RD29A, ATGolS2, ABF3, MYB2, and RD29B, exhibited significantly higher expression in the three OE plants (Fig. 6). However, the expression of six other genes, RbohD, RD22, RAB18, P5CS1, LEA14, and RbohF, were unchanged or slightly increased in the transgenic plants (Fig. 6A). Numerous genes, such as AtNXH1, AtNXH2, AtHKT1, AtCHX16, AtMPK4, AtMPK6, AtSOS1, AtSOS2, and AtSOS3 play roles in coping with and surviving under salt-stress (Park et al. 2016). We also detected the expression of these salinity responsive genes in $\mathrm{OE}$ plants by real-time qPCR and all nine genes were upregulated, especially AtSOS1 and AtSOS3 (the expression of them was more than 2 fold higher than in control plants; Fig. 6B).

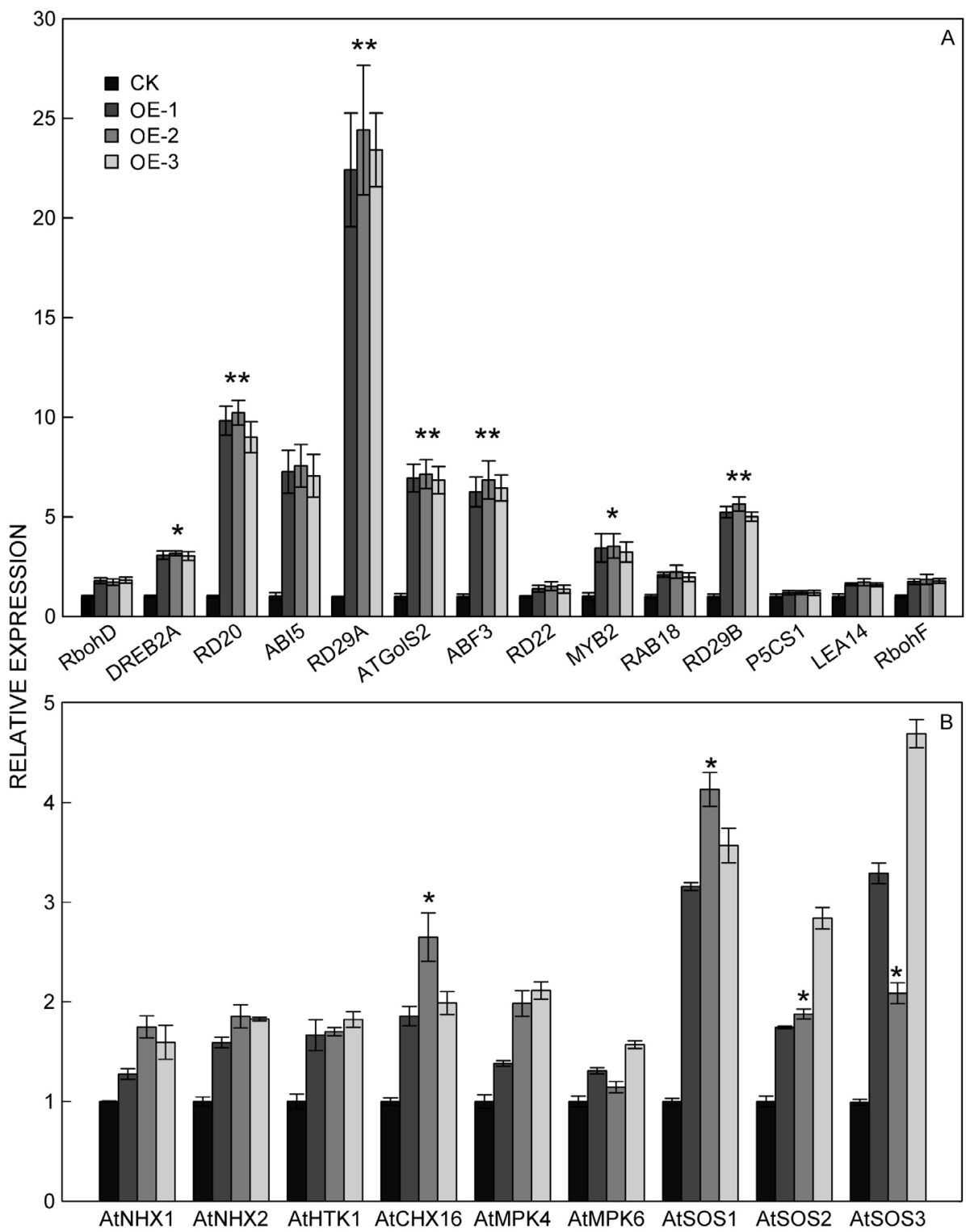

Fig. 6. Expression of stress-responsive genes in control (CK) and ectopic transgenic lines (OE1, OE2, and OE3) of Arabidopsis. RNA was extracted from leaf samples after 30-d growth on $100 \mathrm{mM} \mathrm{NaCl}$ and reverse transcribed to synthesize cDNA. The Actin gene was used as an internal control. $A$ - Expression pattern of fourteen genes induced by both $\mathrm{NaCl}$ and PEG. $B$ - Expression pattern of nine known salt stress responsive genes. Means $\pm \mathrm{SDs}, n=3$, * and $* *$ - significant differences between CK and transgenic lines $0.01<P<0.05$ and $P<0.01$, respectively (Student's $t$-test). 


\section{Discussion}

The important roles of MYB TFs in stress tolerance have been demonstrated in model plants and many crops. In maize, previous studies identified 157 R2R3-MYB TFs. A comprehensive computational analysis was performed to yield an overview of the $R 2 R 3-M Y B$ gene family in maize, including the phylogeny, expression patterns, and structural and functional characteristics (Du et al. 2012), but the expression patterns under different stress treatments were not explored. Here, we focused on the $M Y B$ genes having putative abiotic stress-responsive functions. Using a comparative analysis, we identified $46 M Y B$ genes that are orthologs of stress-resistance $M Y B$ genes from other species, including Arabidopsis, rice, and wheat. In the phylogenetic tree, the ZmMYB genes that share the same clade may have similar functions to the homologous proteins of other species.

The expression patterns of all 46 maize $M Y B$ genes under different abiotic stress conditions were studied. In total, 22 genes that responded to different stress treatments were discovered, of which 16 responded to at least two treatments, indicating that they were major factors involved in the cross-talk among different signal transduction pathways in responses to abiotic stresses. Different stress treatments stimulated the expression of the same genes. Four treatments shared three genes, ZmMYB30, ZmMYB36, and ZmMYB95. The PEG treatment, which mimics drought stress conditions, shared nine responsive genes with the $\mathrm{NaCl}$ treatment, and eight genes with the ABA treatment. The $\mathrm{NaCl}$ treatment shared nine responsive genes with the ABA treatment. Of the six genes responding to cold, only ZmMYB53 was unique. The other five genes also responded to other treatments (Table 3 Suppl.). The ABA is an important factor that participates in regulatory networks involved in abiotic stress responses. The signaltransduction pathways are divided into ABA dependent and ABA independent (Shinozaki and YamaguchiShinozaki, 2007). The 14 ABA responsive genes, except ZmMYB71, responded to other stress treatments. Thus, we could preliminary classify the genes that responded to drought, salt, and cold stresses into two types, playing roles in the ABA-dependent or ABA-independent signaltransduction pathways. For example, in the drought

\section{References}

Cai, G., Wang, G., Wang, L., Pan, J., Liu, Y., Li, D.: ZmMKK1, a novel group A mitogen-activated protein kinase kinase gene in maize, conferred chilling stress tolerance and was involved in pathogen defense in transgenic tobacco. - Plant Sci. 214: 57-73, 2014.

Chen, T., Li, W., Hu, X., Guo, J., Liu, A., Zhang, B.: A cotton MYB transcription factor, GbMYB5, is positively involved in plant adaptive response to drought stress. - Plant Cell treatment, ZmMYB49, ZmMYB111, ZmMYB119, and ZmMYB142 may participate in the ABA-independent signalling pathway, while the other nine genes may participate in the ABA-dependent signalling pathway. However, these predictions need further experimental verification.

In this study, 22 maize MYB proteins were demonstrated to be stress-induced proteins. However, the functions of almost all of these proteins remain unknown. ZmMYB30, whose expression was strongly induced by all treatments, was selected for a further functional analysis through an ectopic expression in Arabidopsis thaliana. Because the expression ZmMYB30 could be highly induced by salt treatment, we determined the salt tolerance potential of OE lines. Really, the OE seedlings exhibited an enhanced salt tolerance during germination. This finding suggested that the ZmMYB30 gene is involved in responses to salinity stress, which is in agreement with its expression pattern.

Some parts of the transcriptional regulatory networks in abiotic stress responses have been identified in Arabidopsis (Shinozaki and Yamaguchi-Shinozaki 2007). The investigation of the expression profiles of major stress-responsive genes and salt-responsive genes in these networks could provide important information for uncovering the mechanisms of ZmMYB30 in salt-stress response and tolerance (Park et al. 2016). Our results showed that the expression of eight stress-responsive genes and nine salt-responsive genes were higher in the OE plants. The enhanced salt-stress tolerance of the $\mathrm{OE}$ plants is at least partially due to the increased expression of these genes.

In the phylogenetic tree, the ZmMYB30 gene was clustered in the same clade with Arabidopsis genes AtMYB44 and AtMYB77. It was reported that these two genes have multiple roles in stress response through ABA signalling pathway (Jaradat et al. 2013, Zhao et al. 2014). According to the expression pattern of ZmMYB30 under stress and preliminary results of functional analysis, ZmMYB30 may be a key player in maize stress response. The potential roles of ZmMYB30 will be further investigated in our ongoing study.

Physiol.56: 917-929, 2015.

Du, H., Feng, B.R., Yang, S.S., Huang, Y.B., Tang, Y.X.: The R2R3-MYB transcription factor gene family in maize. PLoS ONE 7: e37463, 2012.

Habben, J.E., Bao, X., Bate, N.J., DeBruin, J.L., Dolan, D., Hasegawa, D., Helentjaris, T. G., Lafitte, R.H., Lovan, N. Mo, H.: Transgenic alteration of ethylene biosynthesis increases grain yield in maize under field drought stress 
conditions. - Plant Biotech. J. 12: 685-693,2014.

Harrison, M.T., Tardieu, F., Dong, Z., Messina, C.D., Hammer, G.L.: Characterizing drought stress and trait influence on maize yield under current and future conditions. - Global Change Biol. 20: 867-878, 2014.

Hoang, X.L.T., Thu, N.B.A., Thao, N.P., Tran, L.S.P.: Transcription factors in abiotic stress responses: their potentials in crop improvement. - In: Ahmad, P., Wani, M.R., Azooz, M.M., Tran, L.S.P.: (ed.): Improvement of Crops in the Era of Climatic Changes. Pp. 337-366. Springer, New York 2014.

Jaradat, M.R., Feurtado, J.A., Huang, D., Lu, Y., and Cutler, A.J.: Multiple roles of the transcription factor AtMYBR1/AtMYB44 in ABA signaling, stress responses, and leaf senescence. - BMC Plant Biol. 13: 1-19, 2013.

Jiang, S., Zhang, D., Wang, L., Pan, J., Liu, Y., Kong, X., Zhou, Y., Li, D.: A maize calcium-dependent protein kinase gene, $Z m C P K 4$, positively regulated abscisic acid signaling and enhanced drought stress tolerance in transgenic Arabidopsis. - Plant. Physiol. Biochem. 71: 112-120, 2013.

Li, C., Ng, C.K.Y., Fan, L.M.: MYB transcription factors, active players in abiotic stress signaling. - Environ. exp. Bot. 114: 80-91, 2015.

Li, H., Gao, Y., Xu, H., Dai, Y., Deng, D., Chen, J.: ZmWRKY33, a WRKY maize transcription factor conferring enhanced salt stress tolerances in Arabidopsis. Plant Growth Regul. 70: 207-216, 2013.

Lobell, D.B., Roberts, M.J., Schlenker, W., Braun, N., Little, B.B., Rejesus, R.M., Hammer, G.L.: Greater sensitivity to drought accompanies maize yield increase in the US Midwest. - Science 344: 516-519, 2014.

Lu, M., Ying, S., Zhang, D.F., Shi, Y.S., Song, Y.C., Wang, T.Y., Li, Y.: A maize stress-responsive NAC transcription factor, ZmSNAC1, confers enhanced tolerance to dehydration in transgenic Arabidopsis. - Plant Cell Rep. 31: 1701-1711, 2012.

Pan, J., Zhang, M., Kong, X., Xing, X., Liu, Y., Zhou, Y., Liu, Y., Sun, L., Li, D.: ZmMPK17, a novel maize group D MAP kinase gene, is involved in multiple stress responses. Planta 235: 661-676, 2012.

Park, H.J., Kim, W.Y., Yun D.J.: A New insight of salt stress signaling in Plant. - Mol. Cells 39: 447-459, 2016.

Shinozaki, K., Yamaguchi-Shinozaki, K.: Gene networks involved in drought stress response and tolerance. - J. exp.Bot.58: 221-227, 2007.

Tamura, K., Stecher, G., Peterson, D., Filipski, A.,Kumar, S: MEGA6: Molecular evolutionary genetics analysis version 6.0. - Mol. Biol. Evol.30:2725-2729, 2013.

Ying, S., Zhang, D.F., Fu, J., Shi, Y.S., Song, Y.C., Wang, T.Y., Li, Y.: Cloning and characterization of a maize bZIP transcription factor, ZmbZIP72, confers drought and salt tolerance in transgenic Arabidopsis. - Planta 235: 253-266, 2012.

Zhang, D., Jiang, S., Pan, J., Kong, X., Zhou, Y., Liu, Y., Li, D.: The overexpression of a maize mitogen activated protein kinase gene (ZmMPK5) confers salt stress tolerance and induces defence responses in tobacco. - Plant. Biol. 16: 558570, 2014.

Zhang, L., Zhao, G., Xia, C., Jia, J., Liu, X., Kong, X.: A wheat R2R3-MYB gene, TaMYB30-B, improves drought stress tolerance in transgenic Arabidopsis. - J. exp. Bot. 63: 58735885, 2012a.

Zhang, Z., Liu, X., Wang, X., Zhou, M., Zhou, X., Ye, X., Wei, X.: An R2R3 MYB transcription factor in wheat, TaPIMP1, mediates host resistance to Bipolaris sorokiniana and drought stresses through regulation of defense- and stressrelated genes. - New Phytol 196: 1155-70, $2012 \mathrm{~b}$.

Zhao, Y., Xing, L., Wang, X., Hou, Y.-J., Gao, J., Wang, P., Duan, C.-G., Zhu, X., Zhu, J.-K.: The ABA receptor PYL8 promotes lateral root growth by enhancing MYB77dependent transcription of auxin-responsive genes. - Sci. Signal. 7: ra53-ra53, 2014.

Zhou, W., Jia, C.G., Wu, X., Hu, R.X., Yu, G., Zhang, X.H., Liu, J.L., Pan, H.Y.: ZmDBF3, a novel transcription factor from maize (Zea mays L.), is involved in multiple abiotic stress tolerance. - Plant mol. Biol. Rep. 34: 353-364, 2016. 